\title{
Exact Counting of Black Hole Microstates
}

\author{
Atish Dabholkar ${ }^{a, b, c *}$ \\ ${ }^{a}$ Department of Theoretical Physics, Tata Institute of Fundamental Research, Mumbai 400005, India \\ ${ }^{b}$ Institute for Theoretical Physics, Department of Physics, Stanford University, Stanford, CA 94305, USA \\ ${ }^{c}$ Stanford Linear Accelerator Center, Stanford University, Stanford, CA 94025, USA
}

(Dated: September 2004)

\begin{abstract}
The exact entropy of two-charge supersymmetric black holes in $N=4$ string theories is computed to all orders using Wald's formula and the supersymmetric attractor equations with an effective action that includes the relevant higher curvature terms. Classically, these black holes have zero area but the attractor equations are still applicable at the quantum level. The quantum corrected macroscopic entropy agrees precisely with the microscopic counting and moreover the entire macroscopic partition function matches the microscopic partition function for an infinite tower of fundamental string states.
\end{abstract}

A distinctive feature of superstring theory is that its spectrum often contains an infinite tower of BPS states in a given topological sector. The first example of such a tower of BPS states was noticed in the perturbative spectrum of toroidally compactified superstring theories $[1,2]$. We will be interested here in the heterotic string compactified on $\mathbf{T}^{4} \times \mathbf{T}^{2}$ where $\mathbf{T}^{4}$ is a 4 -torus in $\{6789\}$ directions and $\mathbf{T}^{2}$ is a 2 -torus which we take to be a product of two circles in the $\{45\}$ directions. Consider now a string state with winding number $w$ along the $x_{5}$ direction. In a given winding sector, there is a tower of BPS states each in the right-moving ground state but carrying arbitrary left-moving oscillations subject to the Virasoro constraint $N_{L}=1-n w$, where $N_{L}$ is the left-moving oscillation number and $n$ is the quantized momentum along $x_{5}[1,2]$. Note that $N_{L}$ is positive and hence a BPS state that satisfies this constraint has negative $n$ for positive $w$ for large $N_{L}$. We will henceforth denote these states by $(n, w)$.

The number of such states is summarized conveniently by a partition function

$$
Z(\beta)=16 \sum d_{N} e^{-\beta N}
$$

where $N \equiv n|w|=N_{L}-1$. The factor of 16 comes from the degeneracy of the right-moving supersymmetric ground state. Since $N_{L}$ is the number operator for the 24 left-moving transverse bosons, the partition function can be readily evaluated

$$
Z(\beta)=\frac{16}{\Delta(q)},
$$

where $\Delta(q)$ is the Jacobi discriminant function with argument $q=\exp (-\beta)$. In terms of the Dedekind eta function $\eta(q)$, the discriminant is given by $\Delta(q)=\eta(q)^{24}$.

The number of states at level $N$ is then given by the inverse Laplace transform

$$
d_{N}=\frac{1}{2 \pi i} \int_{C} d \beta e^{\beta N} \frac{16}{\Delta\left(e^{-\beta}\right)} .
$$

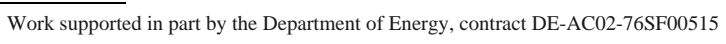

*Electronic address: atish@tifr.res.in
To find the asymptotic density at large $N$, we want to take the high temperature limit or $\beta \rightarrow 0$. It is convenient to use the modular property of the discriminant

$$
\Delta\left(e^{-\beta}\right)=\left(\frac{\beta}{2 \pi}\right)^{-12} \Delta\left(e^{-4 \pi^{2} / \beta}\right) .
$$

As $e^{-4 \pi^{2} / \beta} \rightarrow 0$, we can then use the asymptotics $\Delta(q) \sim$ $q$ and evaluate the integral

$$
d_{N}=\frac{16}{2 \pi i} \int_{C} d \beta e^{\beta N}\left(\frac{\beta}{2 \pi}\right)^{12} \frac{1}{\Delta\left(e^{-4 \pi^{2} / \beta}\right)}
$$

in saddle-point approximation. The saddle point occurs at $\beta=2 \pi / \sqrt{N}$ and the degeneracy has the characteristic exponential growth $d_{N} \sim \exp (4 \pi \sqrt{n|w|})$.

This tower of states has played a crucial role in furthering our understanding of dualities and black hole physics. Heterotic string on $\mathbf{T}^{4} \times \mathbf{T}^{2}$ is dual to Type-IIA on $\mathbf{K}_{3} \times \mathbf{T}^{2}[3,4]$. Initial evidence for this duality came from matching the low-lying BPS states and the supergravity action but a far more stringent test is obtained by matching the entire infinite tower of BPS states. The state $(n, w)$ is dual to $w$ NS5-branes wrapping $\mathbf{K}_{3} \times \mathbf{S}^{1}$ carrying $n$ units of momentum which in turn is dual to $w$ D4-branes wrapping the $\mathbf{K}_{3}$ with a gas $n$ D0-branes on its worldvolume. The characteristic Hagedorn asymptotic density of such brane states was computed in [5]. In fact, this partition function makes its appearance also in topologically twisted Yang Mills theories on $\mathbf{K}_{3}$ which provided one of the early hints of the stringy duality [6]. We will return to this dual description subsequently.

Another important application of this tower of states comes from its relation to black hole entropy. The state $(n, w)$ corresponds to a charged extremal black hole in four dimensions and one would expect that the logarithm of $d_{N}$ for large $N$ should match the Bekenstein-Hawking entropy of this black hole. The corresponding black hole solutions were obtained in [7] which can also be seen to arise directly from the dimensional reduction of the underlying winding string solution in five dimensions [8]. Classically, these black holes have zero area and would appear to have zero entropy but the higher curvature corrections to the supergravity action can correct the solution. Assuming that the string corrections result in a 
finite area horizon it was shown in [9] that the black hole then has nonzero entropy in agreement with the logarithm of the degeneracy. In particular, the nontrivial functional dependence $\sqrt{n|w|}$ on the charges is correctly reproduced. However, the precise numerical factor of $4 \pi$ cannot be computed and the assumption of finite area is in need of further evidence. Subsequent developments have focused mostly on black holes carrying three or more charges that have finite horizon area already classically [10] so that the precise numerical factor can be computed reliably within the supergravity approximation.

In this note we return to the two charge black holes corresponding to the states $(n, w)$ and show that it is possible to take into account exactly all higher curvature corrections to the entropy. After incorporating these corrections and using the exact entropy formula due to Wald [11-13] for the fully corrected action, the precise numerical factor $4 \pi$ in (5) can be computed. One can even go further and in fact reproduce not only the asymptotic density of states for large $N$ but the entire partition function (2) of the whole tower of microscopic states.

Before proceeding further let us note the following curious fact. The curvature squared coupling in the fourdimensional effective action for the heterotic string on $\mathbf{T}^{6}$ is of the form

$$
\frac{1}{16 \pi} \operatorname{Re}\left[\int \frac{\log \Delta(q)}{2 \pi i} \operatorname{tr}\left(R-i R^{*}\right)^{2}\right],
$$

with $q=e^{2 \pi i \lambda}$ where $\lambda$ is the dilaton-axion field, $R$ is the curvature 2 -form and the trace is in the tangent space $S O(1,3)$ representation [14]. The action with its coefficient can be deduced easily from S-duality and GreenSchwarz mechanism. What is striking is that the Jacobi discriminant function that we encountered in (2) makes its appearance here in a completely different context. At first sight this would appear to be little more than a coincidence. After all, the argument $q$ in (6) depends on a spacetime field whereas in (2) it depends on the worldsheet temperature $\beta$. The q-expansion in (6) gives the nonperturbative corrections to the effective coupling from 5-brane instantons [14] whereas the q-expansion in (2) counts the spectrum of perturbative BPS winding states. It turns out, however, that there is indeed a deep and precise connection between the two that is provided by the supersymmetric attractor mechanism [15-17], its elegant implementation in supergravity using Wald's formula for higher derivative F-term corrections pioneered in [18-22], and the recent proposal for the black hole partition function in [23].

Let us summarize the relevant formalism [18-21, 23]. To be closer to the discussion in the literature, we work in the dual description of Type-IIA on $\mathbf{K}_{3} \times \mathbf{T}^{2}$ which can be viewed as a special case of a Calabi-Yau 3-fold. The resulting supergravity in four dimensions has $N=4$ supersymmetry but it will be convenient for our purposes to use the $N=2$ notation of special geometry.

The vector multiplet moduli space of $N=2$ supergravity with $n_{v}$ vector multiplets is parameterized by $n_{v}+$
1 complex projective coordinates $X^{I}, I=0,1, \ldots, n_{v}$. There are an infinite number of higher derivative corrections to the Einstein-Hilbert action that are expected to be relevant for the computation of the entropy. These Ftype corrections to the effective action are summarized by the string-loop corrected holomorphic prepotential

$$
F\left(X^{I}, W^{2}\right)=\sum_{h=0}^{\infty} F_{h}\left(X^{I}\right) W^{2 h}
$$

where $F_{h}$ are computed by the topological string amplitudes [24-28] and $W^{2}$ is the reduced chiral multiplet $[19,29]$ that involves the graviphoton field strength. The prepotential obeys the homogeneity relation

$$
X^{I} \partial_{I} F\left(X^{I}, W^{2}\right)+W \partial_{W} F\left(X^{I}, W^{2}\right)=2 F\left(X^{I}, W^{2}\right) .
$$

The moduli couple to the electromagnetic fields and as a result vary with the radius in the back hole background. Starting with arbitrary values at infinity, at the horizon they approach an attractor point in the moduli space. The values at the attractor point are determined by the black hole attractor equations

$$
\begin{gathered}
p^{I}=\operatorname{Re}\left[C X^{I}\right] \\
q_{I}=\operatorname{Re}\left[C F_{I}\left(X^{I}, \frac{256}{C^{2}}\right),\right]
\end{gathered}
$$

and $C^{2} W^{2}=256$, where $F_{I} \equiv \partial F / \partial X^{I}$ are the holomorphic periods. The scaling field $C$ is introduced so that $\left(C X^{I}, C F_{I}\right)$ is non-projective and transforms like $\left(p^{I}, q_{I}\right)$ as a vector under the $S p\left(2 n_{v}+2 ; \mathbf{Z}\right)$ symplectic duality group. The attractor equations are then determined essentially by symplectic invariance. For a recent review of the leading order attractor equations and their applications see [30].

The quantum corrected black hole entropy is given by [18-21]

$$
S_{\mathrm{BH}}=\frac{\pi i}{2}\left(q_{I} \bar{C} \bar{X}^{I}-p^{I} \bar{C} \bar{F}_{I}\right)+\frac{\pi}{2} \operatorname{Im}\left[C^{3} \partial_{C} F\right]
$$

The first set of attractor equations (9) can be solved by

$$
C X^{I}=p^{I}+\frac{i}{\pi} \phi^{I}
$$

in terms of the 'potentials' $\phi^{I}$. Then the entropy (11) can be written in a suggestive form [23] as

$$
S_{\mathrm{BH}}(q, p)=\mathcal{F}(\phi, p)-\phi^{I} \frac{\partial}{\partial \phi^{I}} \mathcal{F}(\phi, p) .
$$

in terms of a 'free energy' function

$$
\mathcal{F}(\phi, p)=-\pi \operatorname{Im}\left[F\left(p^{I}+\frac{i}{\pi} \phi^{I}, 256\right)\right] .
$$


The potentials $\phi^{I}$ in this equation are determined in terms of the charges by the second set of attractor equations (10)

$$
q_{I}=\frac{1}{2}\left(C F_{I}+\bar{C} \bar{F}_{I}\right)=-\frac{\partial}{\partial \phi^{I}} \mathcal{F}(\phi, p) .
$$

Given the form of the entropy (14), it is natural to define a 'partition function' as suggested in [23]

$$
Z_{\mathrm{BH}}\left(\phi^{I}, p^{I}\right)=e^{\mathcal{F}\left(\phi^{I}, p^{I}\right)} \equiv \sum_{q_{I}} \Omega\left(q_{I}, p^{I}\right) e^{-\phi^{I} q_{I}},
$$

where $\Omega\left(q_{I}, p^{I}\right)$ are the black hole degeneracies. The Boltzmann entropy $\ln \Omega(q, p)$ is then expected to agree with the thermodynamic entropy $S_{\mathrm{BH}}(q, p)$ in (13) for large charges.

It would be very interesting to test the proposal (16) for the black hole partition function by comparing it with the microscopic partition function. For a general CalabiYau compactification, such an explicit comparison is difficult for a number of reasons. On the supergravity side, to make this comparison it is necessary to compute all infinite terms $F_{h}$ in the prepotential (7). Even though these are given in principle by the topological string, they are not always explicitly computable. On the microscopic side, the counting of states is complicated by the fact that the number of BPS states can jump in $N=2$ supersymmetric theories [31]. This phenomenon is possibly related to black hole fragmentation [32] and multiple basins of attraction [33] as suggested in [23]. In addition, there are subtleties having to do with the holomorphic anomalies and the background dependence on hypermultiplet moduli which complicate the picture further[23].

One virtue of the tower of states in $N=4$ compactification that we have considered is that it provides a particularly simple but nontrivial example for making a clean comparison between black hole microstates and the exact entropy formula. For this system, on the macroscopic side the prepotential is explicitly computable. Moreover on the microscopic side the exact partition function of the microstates is also known and is given by (1). With $N=4$ supersymmetry we do not expect that the number of BPS states would jump.

For Type-IIA on a Calabi-Yau manifold, in the large volume limit, $F_{0}$ and $F_{1}$ are given by

$$
F_{0}=-\frac{1}{6} C_{I J K} \frac{X^{I} X^{J} X^{K}}{X^{0}}, \quad F_{1}=-\frac{1}{64} \frac{c_{2}}{24} \frac{X^{1}}{X^{0}},
$$

where $A=1, \ldots, n_{v}$ and $c_{2}$ is the second Chern class and $C_{I J K}$ are the intersection numbers of a basis $\left\{\Sigma^{I}\right\}$ of 4 cycles [29]. For a properly normalized basis of 2 -forms $\left\{\omega_{I}\right\}$ that are Poincare dual to $\left\{\Sigma^{I}\right\}$, the intersection numbers are given by

$$
C_{I J K}=\int_{C Y 3} \omega_{I} \wedge \omega_{J} \wedge \omega_{K} .
$$

In the special case of $\mathbf{K}_{3} \times \mathbf{T}^{2}$, there are 23 2-cycles of which we take $w_{1}$ to be the 2 -torus itself and $w_{a}, a=$
$2, \ldots, 23$ to be the 222 -cycles of $\mathbf{K}_{3}$. The $N=2$ reduction of $N=4$ is a bit subtle in supergravity because of the extra gravitini multiplets. For the particular charge configuration that we have chosen, however, the fields in the gravitini multiplets are not excited. Hence we can safely ignore them.

A major simplification for $\mathbf{K}_{3} \times \mathbf{T}^{2}$ is that in (7), all $F_{h}$ for $h>1$ vanish. This can be seen most easily in the corresponding topological string from the counting of fermion zero modes. Moreover, $F_{0}$ is given by its classical value and receives no quantum corrections because $F_{0}$ determines the metric on the moduli space which is known to receive no corrections in $N=4$ supergravity. Thus, the only nontrivial term in the prepotential comes from $F_{1}$ which has already been computed in the literature in a number of different ways - either directly from its definition [25, 34], or by using the holomorphic anomaly [24], or from string-string duality [14] by requiring agreement with (6). The fully quantum corrected prepotential then takes a particularly simple form

$$
F\left(X, W^{2}\right)=-\frac{1}{2} C_{a b} X^{a} X^{b}\left(\frac{X^{1}}{X^{0}}\right)-\frac{W^{2}}{256 \pi i} \log \Delta(q)
$$

where $q=\exp \left(4 \pi i X^{1} / X^{0}\right)$ and $C_{a b}$ is the intersection matrix of $K 3$ and we have used the fact that $c_{2}=24$ for $\mathbf{K}_{3}$. It can be seen using the action in $[18,35]$ that $2 X^{1} / X^{0}$ is the correctly normalized dilaton-axion field $\lambda$ that couples to integral Pontryagin class so that the action is then invariant under $\lambda \rightarrow \lambda+1$.

Now that we have the exact prepotential, let us see what our charge configuration looks like in this basis. The perturbative state $(n, w)$ on the heterotic side is dual to $w$-branes wrapping the $\mathbf{K}_{3}$ with $n$ 0-branes sprinkled on it. The 4 -cycle is dual to the 2 -form $w_{1}$ and hence we have a nonzero magnetic charge $p_{1}=w$ and all other magnetic charges are zero. The 0-brane couples electrically to the graviphoton field and hence $q_{0}=n$ and all other electric charges are zero. we can readily evaluate the free energy defined in (14)

$$
\mathcal{F}(\phi, p)=-\frac{1}{2} C_{a b} \phi^{a} \phi^{b} \frac{p^{1}}{\phi^{0}}-\log (|\Delta(q)|)
$$

with

$$
q=\exp \left(\frac{4 \pi^{2} p^{1}}{\phi^{0}}+\frac{4 \pi i \phi^{1}}{\phi^{0}}\right) .
$$

In the large volume limit we can approximate the second term in (20) by $-4 \pi^{2} p^{1} / \phi^{0}$. It is then easy to solve the attractor equations for the set of charges $q^{A}=\left(q^{0}, 0,0, \ldots, 0\right)$ and with $p_{A}=\left(0, p_{1}, 0, \ldots, 0\right)$. The solution is

$$
\phi^{0}=-2 \pi \sqrt{\frac{p^{1}}{\left|q_{0}\right|}}, \quad \phi^{a}=0,
$$

and $\phi^{1}$ is undetermined. The entropy is given by

$$
S=4 \pi \sqrt{p^{1}\left|q_{o}\right|}=4 \pi \sqrt{w|n|},
$$


which matches exactly with the logarithm of the degeneracy (5) of the tower of states at large $N$. It is remarkable that once the higher derivative corrections are included, the attractor formalism is powerful enough to correctly reproduce the entropy even for black holes that have zero area classically. This result is implicit in some of the early work [29] however there the focus is on computing the corrections to the entropy of black holes that have finite area already classically.

Encouraged by this, we would now like to reproduce the exact degeneracy $\Omega\left(q^{A}, p_{A}\right)$ in (16) which is given by the inverse Laplace transform,

$$
\left(\frac{1}{2 \pi}\right)^{24} \int \prod_{a=1}^{22} d \phi^{a} d \phi^{1} d \phi^{0} \exp \left(\mathcal{F}\left(\phi^{A}, p^{A}\right)+\phi^{0} q_{0}\right) .
$$

Using the free energy (20), the Gaussian integrals over $\left\{\phi^{a}\right\}$ can be performed immediately to get,

$$
\Omega\left(q_{0}, p^{1}\right) \sim \frac{1}{2 \pi} \int d x\left(\frac{x}{2 \pi}\right)^{12} e^{N x} \int \frac{d \theta}{2 \pi} \frac{1}{|\Delta(q)|},
$$

where we have defined $x=-\phi^{0} / p^{1}$ and $\theta=\phi^{1} / \phi^{0}$ so that $q=\exp 2 \pi i \tau$ with $\tau=2 \theta+2 \pi i / x$ and $N=-p^{1} q_{0} \equiv w|n|$.

We can use the modular property (4) to write the integral as

$$
\Omega\left(q_{0}, p^{1}\right) \sim \frac{1}{2 \pi i} \int d x e^{N x} \frac{1}{\Delta\left(e^{-x}\right)}
$$

Here we have used the approximation $-\frac{1}{\tau} \sim \frac{i}{2 \pi x}$ for small $x$ to perform the $\theta$ integral. We thus see that for large charge with $x=\beta$, the black hole partition function (25) matches with the microscopic partition function of underlying states (3) up to a numerical factor.

\section{Acknowledgments}

I am grateful to Bernard de Wit for valuable discussions and correspondence. I would also like to thank Rajesh Gopakumar for discussions and Andy Strominger for comments on the draft. This work was supported in part by Stanford Institute of Theoretical Physics, the David and Lucile Packard Foundation Fellowship for Science and Engineering, and by the Department of energy under contract number DE-AC02-76SF00515.
[1] A. Dabholkar and J. A. Harvey, Phys. Rev. Lett. 63, 478 (1989).

[2] A. Dabholkar, G. W. Gibbons, J. A. Harvey, and F. Ruiz Ruiz, Nucl. Phys. B340, 33 (1990).

[3] C. M. Hull and P. K. Townsend, Nucl. Phys. B438, 109 (1995), hep-th/9410167.

[4] E. Witten, Nucl. Phys. B443, 85 (1995), hepth/9503124.

[5] C. Vafa, Nucl. Phys. B463, 415 (1996), hep-th/9511088.

[6] C. Vafa and E. Witten, Nucl. Phys. B431, 3 (1994), hepth/9408074.

[7] A. Sen, Nucl. Phys. B440, 421 (1995), hep-th/9411187.

[8] A. Dabholkar, J. P. Gauntlett, J. A. Harvey, and D. Waldram, Nucl. Phys. B474, 85 (1996), hep-th/9511053.

[9] A. Sen, Mod. Phys. Lett. A10, 2081 (1995), hepth/9504147.

[10] A. Strominger and C. Vafa, Phys. Lett. B379, 99 (1996), hep-th/9601029.

[11] R. M. Wald, Phys. Rev. D48, 3427 (1993), grqc/9307038.

[12] V. Iyer and R. M. Wald, Phys. Rev. D50, 846 (1994), gr-qc/9403028.

[13] T. Jacobson, G. Kang, and R. C. Myers (1994), grqc/9502009.

[14] J. A. Harvey and G. W. Moore, Phys. Rev. D57, 2323 (1998), hep-th/9610237.

[15] S. Ferrara, R. Kallosh, and A. Strominger, Phys. Rev. D52, 5412 (1995), hep-th/9508072.

[16] A. Strominger, Phys. Lett. B383, 39 (1996), hepth/9602111.

[17] S. Ferrara and R. Kallosh, Phys. Rev. D54, 1514 (1996), hep-th/9602136.

[18] G. Lopes Cardoso, B. de Wit, and T. Mohaupt, Phys.
Lett. B451, 309 (1999), hep-th/9812082.

[19] G. Lopes Cardoso, B. de Wit, and T. Mohaupt, Fortsch. Phys. 48, 49 (2000), hep-th/9904005.

[20] G. Lopes Cardoso, B. de Wit, and T. Mohaupt, Class. Quant. Grav. 17, 1007 (2000), hep-th/9910179.

[21] G. Lopes Cardoso, B. de Wit, J. Kappeli, and T. Mohaupt, JHEP 12, 019 (2000), hep-th/0009234.

[22] J. M. Maldacena, A. Strominger, and E. Witten, JHEP 12, 002 (1997), hep-th/9711053.

[23] H. Ooguri, A. Strominger, and C. Vafa (2004), hepth/0405146.

[24] M. Bershadsky, S. Cecotti, H. Ooguri, and C. Vafa, Commun. Math. Phys. 165, 311 (1994), hep-th/9309140.

[25] M. Bershadsky, S. Cecotti, H. Ooguri, and C. Vafa, Nucl. Phys. B405, 279 (1993), hep-th/9302103.

[26] I. Antoniadis, E. Gava, K. S. Narain, and T. R. Taylor, Nucl. Phys. B413, 162 (1994), hep-th/9307158.

[27] R. Gopakumar and C. Vafa (1998), hep-th/9809187.

[28] R. Gopakumar and C. Vafa (1998), hep-th/9812127.

[29] G. Lopes Cardoso, B. de Wit, and T. Mohaupt, Nucl. Phys. B567, 87 (2000), hep-th/9906094.

[30] G. W. Moore (2004), hep-th/0401049.

[31] N. Seiberg and E. Witten, Nucl. Phys. B426, 19 (1994), hep-th/9407087.

[32] J. M. Maldacena, J. Michelson, and A. Strominger, JHEP 02, 011 (1999), hep-th/9812073.

[33] F. Denef, JHEP 08, 050 (2000), hep-th/0005049.

[34] L. J. Dixon, V. Kaplunovsky, and J. Louis, Nucl. Phys. B355, 649 (1991).

[35] B. de Wit, Nucl. Phys. Proc. Suppl. 49, 191 (1996), hepth/9602060. 